\title{
Matriciamento em Saúde Mental: práticas e concepções trazidas por equipes de referência, matriciadores e gestores
}

\author{
Matrix support in mental health: practices and concepts \\ brought by reference teams, matrix teams and managers
}

Alexandra Iglesias (https://orcid.org/0000-0001-7188-9650) ${ }^{1}$

Luziane Zacché Avellar (https://orcid.org/0000-0003-3125-2174) ${ }^{2}$

${ }^{1}$ Departamento de Psicologia, Universidade Federal do Espírito Santo (UFES). Avenida Fernando Ferrari 514,

Goiabeiras. 29075-015 Vitória ES Brasil.

leiglesias@gmail.com

${ }^{2}$ Programa de Pós-

Graduação em Psicologia,

Departamento de

Psicologia Social e do

Desenvolvimento, UFES.

Vitória ES Brasil.

\begin{abstract}
The scope of this study was to analyze matrix support in mental health from the practices and conceptions brought by reference teams, matrix teams and managers on the topic. To achieve this, semi structured interviews were conducted with 41 participants, among whom there were 11 managers, 12 reference team professionals from Health Units and 18 matrix team professionals from Psychosocial Care Centers, in addition to 110 hours of observations of matrix practices based on field journal records. The data were then submitted to content analysis. From the information gathered by the participants, two lines of thought were highlighted about the concept of Matrix Support, namely as a forum for productive meetings between health teams on the one hand, and as a strategy of training-on-thejob on the other. These concepts were discussed in juxtaposition with the data brought from the observations of the daily work in practice. Because of this contradiction, it was possible to perceive the need to create, systematize and strengthen spaces for dialog between reference teams, matrix teams and managers, such that the consolidation of matrix support may occur.
\end{abstract}

Key words Matrix support, Mental health, Primary health care, Health management
Resumo Objetivou-se neste estudo analisar o matriciamento em saúde mental a partir das práticas e concepções trazidas pelas equipes de referência, equipes matriciais e gestores a respeito da temática. Para tanto foram realizadas entrevistas semiestruturadas com 41 participantes, dentre eles 11 gestores, 12 profissionais de equipes de referência das Unidades de Saúde e 18 profissionais de equipes matriciais dos Centros de Atenção Psicossocial, além de 110 horas de observações das práticas matriciais com registros em diário de campo. Os dados foram submetidos a análise de conteúdo. A partir das informações trazidas pelos participantes destacaram-se dois grupos de entendimentos sobre Matriciamento: Matriciamento como possibilidade de encontro produtivo entre equipes de saúde e Matriciamento como estratégia de formação em serviço. Tais concepções foram discutidas em contraposição aos dados trazidos das observações do cotidiano desta prática. A partir dai foi possivel apreender a necessidade de criação, sistematização e fortalecimento de espaços de diálogo entre equipes de referência, equipes matriciais e gestores para a consolidação do matriciamento.

Palavras-chave Apoio matricial, Saúde mental, Atenção básica, Gestão em saúde 


\section{Introdução}

Vários autores estudam e destacam a necessidade de integração da saúde mental ao cotidiano das práticas da Atenção Básica para a efetivação da integralidade do cuidado em saúde ${ }^{1-4}$. Neste contexto, o matriciamento em saúde mental surgiu como uma importante estratégia para fazer valer tal articulação, de modo a garantir um cuidado ampliado à saúde, por meio da interação dialógica entre os diversos saberes indispensáveis à produção de saúde. Segundo o Ministério da Saúde ${ }^{5}$, o matriciamento consiste em um arranjo organizacional que visa outorgar suporte técnico -pedagógico em áreas específicas às equipes responsáveis pelo desenvolvimento de ações básicas de saúde para a população.

Assim, o matriciamento se afirma como recurso de construção de novas práticas em saúde mental também junto às comunidades, no território onde as pessoas vivem e circulam, pela sua proposta de encontros produtivos, sistemáticos e interativos entre equipes da Atenção Básica e equipes de saúde mental.

Contudo, a efetivação desta prática enfrenta desafios importantes: mudanças nas relações de trabalho - historicamente hierarquizadas; mudança no modo fragmentado de se operar o cuidado em saúde; mudança na formação em saúde - centrada na perspectiva biomédica; mudança no modo de praticar saúde - que transcende o setor saúde.

Isto significa que a concretização do matriciamento em saúde mental em toda sua potencialidade depende do empenho, disponibilidade e mudanças por parte de todos os envolvidos. Implica negociação entre os diversos saberes presentes, para a construção de diretrizes sanitárias e estratégias de cuidado pertinentes a um determinado contexto $^{6}$. Envolve parceria entre usuários, gestores dos serviços e demais instâncias, equipes responsáveis pelo cuidado longitudinal dos usuários em saúde (equipes de referência), equipes que se agregam às equipes de referência disponibilizando sua prática e conhecimento específico em uma determinada área (equipes matriciais), para a composição de um cuidado integral em saúde.

Neste sentido questiona-se: Quais as possibilidades concretas de consolidação desta prática matricial em toda sua potencialidade? A partir de tal questão, buscou-se analisar neste estudo o matriciamento em saúde mental, a partir das práticas e concepções trazidas pelas equipes de referência, equipes matriciais e gestores a respeito da temática.

\section{Método}

Trata-se de uma pesquisa de abordagem qualitativa, realizada em um município do sudeste brasileiro, o qual se compõe de 29 Unidades Básicas, sendo que 23 trabalham sob a égide da Estratégia de Saúde da Família (ESF), três na lógica da Estratégia de Agentes Comunitários de Saúde (EACS) e três delas Núcleos Ampliados de Saúde da Família (NASF), sem ESF e EACS. As Unidades de Saúde da Família do município são compostas por equipes com médico, enfermeiro, agentes comunitários de saúde e auxiliares de enfermagem. Contam ainda com equipe de Saúde Bucal composta por dentista, técnico de higiene dental e auxiliar de consultório dentário. Além disso, durante o período da pesquisa todas elas também contavam com: assistente social, farmacêutico, fonoaudiólogo, profissional de educação física, psicólogo e sanitarista, alocados na Unidade Básica. Na ocasião da pesquisa o município ainda não contava com Núcleos de Apoio à Saúde da Família (NASF).

Em relação à saúde mental, o matriciamento é realizado pelos três Centros de Atenção Psicossocial - CAPS (CAPS III, CAPS III álcool e drogas e CAPS infanto-juvenil) juntamente às 30 Unidades de Saúde existentes no município. Essa rede conta ainda com médicos psiquiatras em um Centro Municipal de Especialidade e com um hospital da rede estadual, ambos mantem pouca articulação com a Atenção Básica e os CAPS.

Para este estudo foram realizadas entrevistas semiestruturadas com 41 participantes, dentre eles: os três diretores de CAPS, dois coordenadores municipais da área técnica de saúde mental (o coordenador atual e o coordenador da época da implantação do matriciamento no município), seis gerentes de Unidades de Saúde, doze profissionais de equipes de referência das Unidades de Saúde e 18 profissionais de equipes matriciais dos CAPS. Para escolha destas Unidades e dos profissionais entrevistados, a pesquisadora selecionou aqueles com os quais mantinha menor contato no dia a dia de trabalho, já que a pesquisadora, na ocasião era servidora pública do município.

Mediante tais entrevistas, foi possível conhecer o entendimento dos profissionais de saúde em relação às práticas de matriciamento por eles desenvolvidas, bem como compreender as dificuldades e as possibilidades existentes para o seu desenvolvimento.

Além das entrevistas, realizou-se observação dos encontros entre equipes de referência e equipes matriciais em Unidades de Saúde. Ao todo 
foram 36 dias de observação, o que significou 110 horas. A intenção inicial era participar das reuniões regionais, que contemplavam a presença das equipes de referência, das equipes matriciais e da gestão, incluindo a coordenação municipal de saúde mental; no entanto, como tais reuniões foram extintas, foi feita a observação dos encontros matriciais entre profissionais da Atenção Básica e dos CAPS. Essas reuniões aconteciam, em média, uma vez por mês, geralmente, no espaço da própria Unidade, com dois profissionais do CAPS (equipe matricial) de categorias diversas e profissionais da Atenção Básica (equipe de referência) - principalmente, assistente social, psicólogo, agente comunitário de saúde e enfermeiro.

Tanto as entrevistas quanto as observações foram realizadas após consentimento dos participantes, da Secretaria Municipal de Saúde e do Comitê de Ética em Pesquisa.

Para essas observações utilizou-se de roteiro com questões referentes ao modo como os atores presentes se relacionavam e como o matriciamento acontecia em ato. Todas as informações e impressões da pesquisadora foram registradas em diário de campo.

A partir daí, os dados das entrevistas e do diário de campo foram analisados tendo como base a análise de conteúdo de $\operatorname{Bardin}^{7}$, a qual consiste em um conjunto de técnicas de análise das comunicações que visa obter indicadores pertinentes à inferência de conhecimentos relativos às condições de produção/recepção destas mensagens. Para a análise foram consideradas as falas como um relato do "grupo equipe de referência", do "grupo equipe matricial" e do "grupo gestores".

\section{Resultados e discussões}

Como resultado destacou-se dois principais grupos de entendimentos: Matriciamento como possibilidade de encontro produtivo entre equipes de saúde e Matriciamento como estratégia de formação em serviço, que serão descritos e discutidos com base na literatura da área. As categorias de análise serão ilustradas com algumas falas/ recortes das entrevistas e dos diários de campo, escolhidas por serem representativas da ideia central da categoria.

\section{Matriciamento como possibilidade de encontro produtivo entre equipes de saúde}

$\mathrm{Na}$ análise das entrevistas dos três grupos de entrevistados, foi marcante a associação do ma- triciamento à possibilidade de garantia de encontro produtivo entre equipes da Atenção Básica e equipes dos CAPS, com vistas à ampliação do cuidado em saúde mental. Neste sentido, foi trazido nas entrevistas a possibilidade desta estratégia de trabalho fomentar a integralidade, reabilitação psicossocial, corresponsabilidade do serviço com o cuidado (grupo gestores).

O resultado da concepção mencionada anteriormente é a afirmação de que houve melhoria no diálogo, inclusive sobre os medos que alguns profissionais das Unidades de Saúde sentiam em lidar com pessoas em sofrimento psíquico. Eu vi que o CAPS se aproximou das discussões a respeito do território e a US da saúde mental (grupo gestores). Vejo que depois que começou a ter o apoio matricial, de se encontrar mesmo, mais profissionais na Unidade começaram a se envolver na saúde mental, além do psicólogo, ainda não é o ideal (grupo equipe de referência). Tinha aquele medo, ainda tem, falou 'saúde mental', 'aí meu Deus', mas mudou, CAPS e Unidade conversam (grupo equipe matricial).

A concepção de que o matriciamento consiste no encontro produtivo entre equipes de saúde é defendida por vários autores que tratam deste assunto $^{8-13}$. Segundo Bertussi ${ }^{11}$, o matriciamento se refere a essa criação de espaços de encontros favoráveis ao diálogo e à pactuação; no caso da saúde mental, principalmente com vistas à ampliação das possibilidades de fomento de um cuidado integral à pessoa em sofrimento psíquico, por meio do envolvimento de vários serviços, setores e atores na empreitada da desinstitucionalização e promoção à saúde.

Contudo, as análises das entrevistas e das observações revelam também, a predominância de certa aspereza nas relações entre os profissionais e gestores envolvidos, bem como a pouca presença dos gestores neste trabalho, como se o matriciamento fosse unicamente responsabilidade dos matriciadores dos CAPS e necessário apenas às equipes da Atenção Básica. O matriciamento foi implantado e meio que deixado por conta dos CAPS capacitarem a Atenção Básica; é importante para os dois. Agora é com eles (grupo de gestores).

Isto pode apontar certa confiança por parte da gestão nos profissionais da atenção básica e dos CAPS em trabalharem na lógica do matriciamento, mas também sinaliza pouco envolvimento e certo desconhecimento das responsabilidades dos gestores com esta proposta matricial; principalmente ao considerar a extinção, por essa gestão, dos espaços de encontros, existentes neste município, entre matriciadores, gestores e equi- 
pes de referência ${ }^{14}$, unanimemente destacados pelos entrevistados como importante espaço para a consolidação do matriciamento. Mantiveram-se os encontros entre as equipes matriciais e as de referência, que ocorrem nos espaços das Unidades, geralmente para a discussão de caso. Certamente trata-se de um espaço importante para a consolidação do matriciamento, mas que não contempla a participação dos gestores para algumas deliberações necessárias relativas ao processo matricial, como, por exemplo, a garantia de horário na agenda dos profissionais para a efetivação de ações conjuntas entre os dois serviços.

Vale destacar que apenas em um único encontro de quatro horas - das 110 horas de observação realizada - o diretor de um dos CAPS e o diretor de uma Unidade estiveram presentes para mediarem uma tensão existente entre equipe da Atenção Básica e equipe matricial do CAPS. Neste momento o diretor do CAPS proferiu uma palestra para falar de suas experiências bem sucedidas neste trabalho interdisciplinar.

Neste caso a tensão em si não foi discutida nessa reunião, prevaleceram nos encontros subsequentes reuniões burocráticas de encaminhamentos dos casos e das situações envolvendo a temática da saúde mental. Com falas do tipo: Vocês do CAPS querem empurrar mais isso para a gente, saúde mental é com vocês (grupo equipe de referência).

Manteve-se claramente o distanciamento entre as duas equipes (equipe da Atenção Básica e equipe do CAPS), ao ponto de alguns profissionais da Unidade de Saúde sentarem fora da roda de discussão, em algumas dessas reuniões que participamos, e se recusarem a integrar o debate sobre um determinado caso, por considera-lo repetitivo e desnecessário, acrescentando não entenderem porque os manicômios deixaram de existir, era só humanizar mais (grupo equipe de referência). Apesar dos incômodos gerados por esta fala, inclusive apontados depois por vários profissionais nos corredores dos serviços, tais divergências, mais uma vez, não foram discutidas. Tudo isso gerou um espaçamento ainda maior entre uma reunião de matriciamento e outra, ao invés de uma intensificação dos encontros, que permitissem trabalhar estas divergências de concepções tão caras à Reforma Psiquiátrica ${ }^{15-17}$.

A Reforma Psiquiátrica Brasileira comparece justamente, como um processo de luta pela construção contínua de reflexão e transformação nos campos assistencial, cultural e conceitual, com vistas à mudança das relações que a sociedade, os sujeitos e as instituições estabeleceram com as pessoas que vivenciam o sofrimento psíquico, baseadas, grande parte das vezes, no estigma, na segregação e na desqualificação desses sujeitos $^{18}$. Requisita, assim, a inserção da pessoa em sofrimento psíquico como sujeito de direito, que como qualquer indivíduo deve ter seu acesso assegurado em todos os níveis de atenção, inclusive e principalmente na Atenção Básica.

Nesse sentido, como nos trazem autores nacionais e internacionais ${ }^{6,19-21}$, que tratam da descentralização da atenção em saúde mental e desestigmatização do sofrimento psíquico, a consolidação das propostas trazidas pela Reforma Sanitária e pela Reforma Psiquiátrica - sustentáculos do matriciamento - requerem uma garantia de sistematicidade e continuidade dos encontros entre a saúde mental e a Atenção Básica. Além de tal regularidade desses encontros, eles devem estar abertos à crítica, ao confronto de ideias, ao questionamento das realidades produzidas, a fim de evitar qualquer cristalização do processo e favorecer as transformações necessárias à promoção à saúde. Como nos traz Figueiredo e Onocko-Campos ${ }^{22}$, o matriciamento necessita ser realimentado constantemente por espaços de reflexão e formação permanente, para que não se perca em uma prática hierarquizada $\mathrm{e}$ especializada baseada em procedimentos.

Como nos traz Bertussi ${ }^{11}$, a intenção com estes encontros não deve ser pôr fim as disputas, conflitos e tensões existentes nestas relações, mas de colocá-los em outro patamar que possibilite, justamente, diálogos abertos à negociação e a instituição de outros modos destes atores se relacionarem, outras possibilidades de organização da gestão do cuidado em saúde, outros processos de trabalho implicados com a vida individual e coletiva da população como um todo.

Para exemplificar: uma situação que chamou atenção e mobilizou Unidade de Saúde e CAPS foi o caso de um senhor em ideação suicida e uso prejudicial de álcool, que acabou vindo a óbito por complicações no seu estado geral de saúde. Apesar das diferenças de entendimentos entre gestores, matriciadores e equipes de referência a respeito da condução do caso - presentes nos relatos das entrevistas - estas questões não foram colocadas em discussão em nenhuma reunião matricial, ao contrário, um ficou atribuindo ao outro a responsabilidade por possíveis equívocos desta condução.

A partir da análise das entrevistas observouse que: para o gestor, o caso foi mal sucedido porque os matriciadores dos dois CAPS não conseguiram se integrar para garantir a regularidade 
dos acompanhamentos pelos dois serviços ao usuário e assegurar que a Unidade mantivesse visitas domiciliares sistemáticas e atendimento médico para revisão do quadro clínico da pessoa em questão. A equipe de referência da Atenção Básica, por sua vez, também percebeu o caso como sendo mal sucedido por entender que a equipe matricial deveria ter inserido o usuário em atenção diária no CAPS e a gestão deveria se posicionar com critérios claros a respeito da atenção em saúde mental. A equipe matricial, por fim, analisou o caso como bem sucedido por considerar que foi possível envolver muitas pessoas neste cuidado ao usuário, mesmo que depois ele tenha vindo a óbito por complicações no seu quadro geral de saúde. De qualquer modo, essa equipe matricial acrescentou: é que a Atenção Básica também poderia ter se empenhado mais no acompanhamento desse caso (grupo equipe matricial).

Como nos diz Campos $^{23}$ : ao discutir as diferenças de opiniões sobre uma mesma situação: quem estaria com a "verdade"? A partir da contraposição das informações trazidas das entrevistas com os dados das observações, pode se dizer que todas as perspectivas são parcialmente "verdadeiras". Contudo, o que não houve foi o encontro produtivo destes pontos de vistas para a construção conjunta das transformações necessárias a este contexto. Desta forma, corrobora-se a afirmativa de Campos ${ }^{23}$ sobre a importância da consolidação de espaço coletivo, que implique em "análise, discussão, interpretação, deliberação, contrato conjunto e definição de tarefa”, para que esta diferença de percepção não "vire guerra civil, porque, se virar guerra civil” é o sistema único de saúde quem perde. $\mathrm{O}$ autor ${ }^{23}$ destaca estes espaços dialógicos como capazes de construir uma compreensão complexa das situações. Acrescenta-se ainda a capacidade desses encontros dialógicos, clarificar as responsabilidades de cada um neste processo, que assim, possivelmente, terá força para diminuir as asperezas das relações e consequentemente, viabilizar a proposta do SUS e a perspectiva de integração do matriciamento.

Neste sentido, foi possível acompanhar também, encontros matriciais marcadamente favoráveis à promoção à saúde e a desinstitucionalização, reconhecidos inclusive pelos envolvidos como conquista de uma prática diferenciada em saúde (grupo equipe de referência). A partir da integração matricial com construção compartilhada de projetos terapêuticos singulares (PTS), foi possível promover uma maior circulação pelo território, de pessoas que antes não eram percebidas como parte daquela comunidade. Merece destaque o caso de um jovem em uso prejudicial de drogas, a articulação matricial entre Unidade de Saúde, CAPS, CRAS e o próprio usuário, resultou na construção de um projeto terapêutico singular, o qual possibilitou uma mudança no modo da comunidade perceber esse jovem e consequentemente uma maior circulação do mesmo pelo território. O processo de acompanhamento e cuidado desta pessoa acabou por incentivá-la a se inserir em curso profissionalizante na área de panificação e ser reconhecida pela comunidade por suas habilidades na cozinha. Este jovem voltou a estudar e retomou seu emprego, continua a fazer o uso de drogas, porém, as pessoas não o veem mais como o drogado do bairro (grupo equipe matricial).

Pode-se considerar tal intervenção como uma ação voltada à promoção, à saúde e à desinstitucionalização, na medida em que se afirma o sujeito como corresponsável por sua vida, capaz de junto a outros atores sociais, inventar outros modos, outras posturas e outras formas de estar no mundo. Além disso, essa intervenção opera também pela desinstitucionalização, no momento em que avança no sentido de mudança no modo da comunidade acolher e enxergar esta pessoa em sofrimento.

\section{Matriciamento como estratégia de formação em serviço}

Foi marcante também a associação por parte dos gestores, matriciadores e equipes de referência do matriciamento à possibilidade de formação em serviço. Eu entendo que no matriciamento uma equipe que está diante de um caso complexo solicita o apoio para quase que supervisionar o caso e dar orientação a esta equipe para ação, como uma formação mesmo (grupo gestores). O apoio tem a ver com uma capacitação técnica-pedagógica para a equipe aqui também cuidar do paciente com transtorno mental (grupo equipe de referência). A gente vê o matriciamento como uma formação em serviço, em que nós e a atenção básica apreendemos um com o outro, encontro, entende? (grupo equipe matricial).

Tal concepção está ligada à conceituação trazida por Campos e Domitti ${ }^{8}$ e pelo Ministério da Saúde ${ }^{5}$, que anunciam a função de suporte técnico-pedagógico do matriciamento às equipes que prestam atenção aos problemas de saúde da população.

Os entrevistados afirmaram o matriciamento como uma possibilidade de formação voltada à troca, inclusive um matriciador fala em entrevis- 
ta da perspectiva de alternância de papéis entre equipe de referência e equipe matricial, uma vez que entende que todos ensinam e aprendem nesta relação matricial (grupo equipe matricial).

Estas informações contrastam em certo ponto os dados provenientes da análise dos diários de campo, uma vez que prevaleceu em ato uma relação formativa em que o matriciador transmite o que deve ser feito pela equipe de referência; ou quando considera que o caso tem indicação para o CAPS, o encaminha para aquele serviço, inclusive é isto que por vezes esperam as equipes de referência também. Eu penso que o CAPS vem aqui para triar quem vai para lá, e vou te falar, dá um alivio quando vai para lá, menos um; é só expectativa (grupo equipe de referência).

Quando os encontros matriciais estão focados na transmissão de informação do matriciador à equipe de referência, geralmente se estrutura relações marcadamente verticalizadas, inclusive tal percepção é trazida também por gestores, matriciadores e equipes de referência nas entrevistas, como se apenas a Atenção Básica tivesse algo a aprender e os matriciadores a ensinar. $O$ apoio na verdade é para dizer o que tem que fazer, fiscalizar, cobrar, aí ficam dificiceis as relações, por que são profissionais do mesmo nível, só de serviços diferentes (grupo gestores).

Neste sentido, é possível deixarem escapar momentos importantes de vinculação e discussões envoltas ao cuidado em saúde mental, por entenderem que a função do matriciamento se limita a transmissão de informação. Como observado em um registro do diário de campo: a médica de família falava, em uma reunião matricial, de sua angústia diante do pouco tempo para atender uma pessoa em intenso sofrimento psíquico. Na fala da médica: Assim fica difícil, a gente tem que lutar para fazer Estratégia de Saúde da Família, porque se não luta a gente fica fingindo que está atendendo.

Nesta situação, a equipe matricial presente nesta reunião solicitou que voltassem para a discussão de caso, perdendo a oportunidade de construir uma parceria e vinculo com aquela equipe. Desta forma, a situação-problema trazida não foi agarrada como uma possibilidade de efetiva problematização para a construção de relações de maior entrosamento destas equipes e de alternativas viáveis de resolução da situação ${ }^{24}$.

Por outro lado, por vezes, também foi possível observar equipes de referência em posição de interpretar todas as atitudes da equipe matricial como falta de disponibilidade, seja quando a equipe matricial solicitava um aprofundamento de discussão sobre um determinado caso, seja em sua ação de impedimento de livre fluxo ao especialista, que se apresenta como uma função do matriciamento ${ }^{25}$ para a construção de um cuidado integral em saúde.

Neste contexto, cada um dos profissionais tende a se fixar no seu núcleo de saber, em uma relação com o saber voltada à definição e afirmação de fronteiras rígidas. Assim, qualquer opinião ou crítica que toca o núcleo de saber do outro é percebida como descabida e invasiva, prevalecendo "um imaginário de mútua exclusão e competitividade"26 entre equipe de referência e matriciadores. Isso dificulta sobremaneira o compartilhamento de saberes e práticas pretendidos pelo matriciamento para a construção conjunta de um cuidado integral em saúde.

Dessa forma, não foi possível observar efetivamente aquela possibilidade de alternância de papéis entre equipes de referência e matriciadores, conforme anunciado acima por um matriciador. As funções em grande parte se mantiveram rígidas: o matriciador/especialista - que tem o conhecimento em saúde mental - deve dar as instruções e a equipe de referência - desprovida deste conhecimento específico - assimilar este treinamento.

Diante destas discussões, faz-se indispensável encarar este processo formativo e o diálogo sobre os pressupostos do matriciamento e seus objetivos como uma necessidade de aprofundamento por todos os envolvidos neste trabalho, uma vez que apesar de gestores, matriciadores e equipes de referências trazerem em algumas entrevistas conceituações próximas ao que teoricamente tem sido discutido sobre matriciamento, em ato foi possível observar ainda práticas na contramão à complexidade da proposta matricial, como, por exemplo, a subutilização dos recursos matriciais - geralmente restritos à discussão superficial de caso; equipes de referência fixas na ideia de encaminhamento; gestores que desconhecem as pretensões do matriciamento; matriciadores com insuficiente identificação com a proposta, desconhecimento do que seja matriciamento e pouco manejo das tecnologias relacionais - que devem permear este processo de trabalho.

O matriciamento prima pela possibilidade de aprender a aprender inclusive com as críticas, com as dúvidas, com os equívocos, com o processo, por uma relação mais transversalizada de saber/poder ${ }^{27}$. Nisto está implicada certa perda de identidade profissional, de status, de ambientes profissionais e modos de trabalho familiares, o que reafirma a necessidade de uma proposta de formação em serviço sistemática e longitudinal 
envolvendo gestores, equipes de referência e matriciadores, para que as possíveis resistências a tantas mudanças não se revertam em prática que, apesar de carregar o nome de matriciamento em saúde mental, caminhe na direção contrária aos princípios do SUS, à Política de Atenção Básica, à Reforma Psiquiátrica ${ }^{6}$.

\section{Considerações finais}

A análise do matriciamento a partir das concepções trazidas pelas equipes de referência, equipes matriciais e gestores a respeito da temática, possibilitou perceber mudanças importantes no cuidado em saúde mental. A partir do matriciamento essas transformações ocorreram tanto para os CAPS - que se aproximaram das discussões a respeito do território (grupo gestores) - quanto para as Unidades de Saúde, as quais se apropriaram da temática da saúde mental. Destacam-se ainda a redução dos medos, principalmente de profissionais das Unidades de Saúde, em lidar com as pessoas em sofrimento psíquico e a aproximação entre Unidades Básicas de Saúde e CAPS, em uma troca produtiva de saberes e práticas.

Desta forma, foi possível acompanhar encontros matriciais marcadamente favoráveis à promoção à saúde e a desinstitucionalização, reconhecidos como conquista de uma prática diferenciada em saúde (grupo equipe de referência). Ressalta-se a maior circulação pelo território, de pessoas que antes não eram percebidas como parte de uma determinada comunidade. São visíveis, então, os efeitos decorrentes desses encontros produtivos para usuários, trabalhadores e sistema de saúde como um todo.

Por outro lado, principalmente no que se refere à análise das práticas a partir das observações de campo, foi possível apreender certa aspereza nas relações entre profissionais e gestores envolvidos, em um movimento de nomeação de culpados em relação a um caso, por exemplo, entendido por alguns com mal sucedido. Destacou-se ainda certo desconhecimento das responsabilidades de cada um desses atores - equipe de referência, equipe matricial e gestores - no matriciamento, com pouco envolvimento da gestão nesse processo, como se o matriciamento fosse unicamente responsabilidade dos matriciadores dos CAPS e necessário apenas às equipes da Atenção Básica.

Nesse contexto, foram extintos os espaços de encontros, existentes neste município, entre matriciadores, gestores e equipes de referência, re- duzindo ainda mais a possibilidade de discussão e análise das tensões existente nessa relação matricial. As funções - equipe de referência e equipe matricial - por vezes se mantiveram rígidas: os matriciadores [...] para dizer o que tem que fazer, fiscalizar, cobrar [...] e a equipe de referência para assimilar a orientação.

Diante dessas análises, este estudo reforça a necessidade de criação, sistematização e fortalecimento de espaços de diálogo entre equipes de referência, equipes matriciais e gestores, para a consolidação do matriciamento. O matriciamento em saúde mental, com todos os desafios que carrega, depende de um processo de construção de modos de se relacionar mais integrados, que não cabe em encontros eventuais ou esporádicos. Inclusive nessa proposta matricial cabe a discussão daquelas tensões e dos processos de trabalho desses serviços envolvidos.

Como nos traz Bastos $^{28}$, o matriciamento envolve um transformar-se e ofertar-se ao outro, para a promoção coletiva de saúde em conformidade às politicas públicas. Desta forma, pode-se dizer que não basta implantar o matriciamento se certas garantias não acompanharem este trabalho, como aqueles encontros produtivos, regulares e longitudinais, que envolvam equipes de referência, matriciadores e gestores. Nisso os gestores têm uma grande responsabilidade, se afirmando como também imprescindíveis na consolidação do matriciamento. A partir daí é possível um processo de efetivação da proposta matricial de formação em serviço, construção de um trabalho interdisciplinar, descentralização da saúde mental e, consequentemente, de integração da pessoa em sofrimento psíquico como sujeito de direito.

Por tudo isto, reforça-se a necessidade de formação nesta perspectiva matricial, análise e acompanhamento constante desta prática, para que em nome do matriciamento em saúde mental não se reproduzam práticas que carregam apenas um nome politicamente aceito e valorizado, sem se sustentar no que ele traz de mais importante para a consolidação dos princípios doutrinários do SUS: a transformação das relações no sentido de maior compartilhamento para as transformações sociais.

\section{Colaboradores}

A Iglesias trabalhou na concepção, pesquisa e redação final e LZ Avellar trabalhou na concepção e revisão crítica do trabalho. 


\section{Referências}

1. Lancetti A. Saúde mental e Saúde da Família. In: Lancetti A, organizador. Saúde Loucura. 2a ed. São Paulo: Hucitec; 2001. p. 117-120.

2. Souza AC. Em tempos de PSF: novos rumos para atenção em Saúde Mental? [dissertação]. Rio de Janeiro: Fiocruz; 2004.

3. Tanaka OY, Lauridsen-Ribeiro E. Desafio para a Atenção Básica: incorporação da assistência em saúde mental. Cad Saude Publica 2006; 22(9):1845-1853.

4. Onocko-Campos R, Gama CA, Ferrer AL, Santos DVD, Stefanello S, Trapé TL, Porto K. Saúde mental na Atenção Primária à Saúde: um estudo avaliativo em uma grande cidade brasileira. Cien Saude Colet 2011; 16(2):4643-4652.

5. Brasil. Ministério da Saúde (MS). Saúde Mental e Atenção Básica: o vínculo e o diálogo necessários. In: Brasil. MS. Saúde Mental no SUS: os centros de atenção psicossocial. Brasília: MS; 2003. p. 77-84.

6. Hirdes A, Silva MKR. Apoio matricial: um caminho para a integração saúde mental e atenção primária. Saúde Debate 2014; 38(102):582-592.

7. Bardin L. Análise de conteúdo. Lisboa: Ed. 70; 1988.

8. Campos GWS, Domitti AC. Apoio Matricial e equipe de referência: uma metodologia para gestão do trabalho interdisciplinar em saúde. Cad Saude Publica 2007; 23(2):399-407.

9. Bezerra E, Dimenstein M. Os CAPS e o trabalho em rede: tecendo o Apoio Matricial na Atenção Básica. Psicol, Cienc Prof 2008; 28(3):632-645.

10. Dimenstein M, Severo AK, Brito M, Pimenta AL, Medeiros V, Bezerra E. O Apoio Matricial em Unidades de Saúde da Família: experimentando inovações em Saúde Mental. Saude Soc 2009; 18(1):63-74.

11. Bertussi DC. O Apoio Matricial rizomático e a produção de coletivos na gestão municipal em saúde [tese]. Rio de Janeiro: Universidade Federal do Rio de Janeiro; 2010.

12. Morais APP, Tanaka OY. Apoio matricial em saúde mental: alcances e limites na Atenção Básica. Saúde Soc 2012; 21(1):161-170.

13. Minozzo F, Da Costa II. Apoio matricial em saúde mental: fortalecendo a saúde da família na clínica da crise. Rev Latinoamericana de Psicopatologia Fundamental 2013; 16(3):438-450.

14. Iglesias A, Avellar LZ. Matrix Support in mental health: The experience in Vitória, Espírito Santo. J Health Psychol 2016; 21(3):346-355.

15. Amarante P. Novos sujeitos, novos direitos: o debate em torno da Reforma Psiquiátrica. Cad Saude Publica 1995; 11(3):491-494.

16. Amarante P. Loucos pela vida. Trajetória da reforma psiquiátrica no Brasil. Rio de Janeiro: Fiocruz; 1998.

17. Alverga AR, Dimenstein M. A reforma psiquiátrica e os desafios na desinstitucionalização da loucura. Interface Botucatu 2006; 10(20):299-316.
18. Amarante P. Loucura, cultura e subjetividade: conceitos e estratégias, percursos e atores da reforma psiquiátrica brasileira. In: Fleury S, organizador. Saúde e Democracia: a luta do CEBES. São Paulo: Lemos Editorial; 1997. p.163-186.

19. Nicácio MF. Utopia da realidade: contribuições da desinstitucionalização para a invenção de serviços de Saúde Mental [tese]. Campinas: Universidade Estadual de Campinas; 2003.

20. Patel V, Maj M, Flisher AJ, De Silva MJ, Koschorke M, Prince M; WPA Zonal and Member Society Representatives. Reducing the treatment gap for mental disorders: a WPA survey. World Psychiatry 2010; 9(3):169176.

21. Minoletti A, Rojas G, Horvitz-Lennon M. Salud mental en atención primaria en Chile: aprendizajes para Latinoamérica. Cad Saude Colet, 2012; 20(4):440-447.

22. Figueiredo MD, Campos RO. Saúde Mental e Atenção Básica à Saúde: o Apoio Matricial na construção de uma rede multicêntrica. Saúde Debate 2009; 32(79):143-149.

23. Campos GWS. Apoio matricial e práticas ampliadas e compartilhadas em redes de atenção. Psicol Rev 2012; 18(1):148-168.

24. Oliveira GN. Apoio Matricial como Tecnologia de Gestão e Articulação em Rede. In: Campos GW S, Guerreiro AVP, organizadores. Manual de Práticas de Atenção Básica: saúde ampliada e compartilhada. São Paulo: Hucitec; 2008. p. 154-178.

25. Figueiredo MD. Saúde Mental na Atenção Básica: Um estudo hermenêutico-narrativo sobre o Apoio Matricial na rede SUS-Campinas (SP) [dissertação]. Campinas: Universidade Estadual de Campinas; 2006.

26. Cunha GT, Campos GWS. Apoio matricial e Atenção Primária em Saúde. Saúde Soc 2011; 20(4):961-970.

27. Iglesias A. O Matriciamento em Saúde Mental sob vários olhares [tese]. Vitória: Universidade Federal do Espírito Santo; 2015.

28. Bastos ENE. A prática cotidiana e a função apoiadora. In: Seminário Nacional de Humanização do SUS, 2, 2009, Brasília. Anais do II Seminário Nacional de Humanização: Trocando Experiências. Aprimorando o SUS, 2009. [acessado 2016 Out 28]. Disponível em: http://www.sispnh.com.br/anais/inicio.asp

Artigo apresentado em 24/11/2016

Aprovado em 27/06/2017

Versão final apresentada em 29/06/2017 